Algebraic 85 Geometric Topology

Volume 3 (2003) 593-622

ATG

Published: 22 June 2003

\title{
Plane curves and their fundamental groups: Generalizations of Uludağ's construction
}

\author{
DAVID GARBer
}

\begin{abstract}
In this paper we investigate Uludağ's method for constructing new curves whose fundamental groups are central extensions of the fundamental group of the original curve by finite cyclic groups.

In the first part, we give some generalizations to his method in order to get new families of curves with controlled fundamental groups. In the second part, we discuss some properties of groups which are preserved by these methods. Afterwards, we describe precisely the families of curves which can be obtained by applying the generalized methods to several types of plane curves. We also give an application of the general methods for constructing new Zariski pairs.
\end{abstract}

AMS Classification 14H30; 20E22,20F16,20F18

Keywords Fundamental groups, plane curves, Zariski pairs, Hirzebruch surfaces, central extension

\section{Introduction}

The fundamental group of complements of plane curves is a very important topological invariant with many different applications. This invariant was used by Chisini [8], Kulikov [14] and Kulikov-Teicher [15] in order to distinguish between connected components of the moduli space of surfaces of general type. Moreover, the Zariski-Lefschetz hyperplane section theorem (see [16]) showed that

$$
\pi_{1}\left(\mathbb{P}^{N}-S\right) \cong \pi_{1}(H-H \cap S)
$$

where $S$ is an hypersurface and $H$ is a generic 2-plane. Since $H \cap S$ is a plane curve, this invariant can be used also for computing the fundamental group of complements of hypersurfaces in $\mathbb{P}^{N}$.

A different direction for the need of fundamental groups' computations is for getting more examples of Zariski pairs [36, 37]. A pair of plane curves is called 
a Zariski pair if they have the same combinatorics (i.e. the same singular points and the same arrangement of irreducible components), but their complements are not homeomorphic. Several families of Zariski pairs were presented by ArtalBartolo [3, 5], Degtyarev [9], Oka [20] and Shimada [26, 27, 28]. Tokunaga and his coauthors thoroughly investigated Zariski pairs of curves of degree 6 (see $[6,7,29,30]$ and [31]). Some candidates for weak Zariski pairs (where we take into account only the types of the singular points) can also be found in [13], where any pair of arrangements with the same signature but with different lattices can serve as a candidate for a Zariski pair (it is only needed to be checked that the pair of arrangements have non-isomorphic fundamental groups).

It is also interesting to explore new finite non-abelian groups which are serving as fundamental groups of complements of plane curves.

Uludağ $[32,33]$ presents a way to obtain new curves whose fundamental groups are central extensions of the fundamental group of a given curve. Using his method, one can produce a family of examples of Zariski pairs from a given Zariski pair (see also Section 6 here). His main result is:

Theorem 1.1 (Uludağ) Let $C$ be a plane projective curve and $G=\pi_{1}\left(\mathbb{P}^{2}\right.$ $C)$. Then for any $n \in \mathbb{N}$, there is a curve $\tilde{C} \subset \mathbb{P}^{2}$ birational to $C$ such that $\tilde{G}=\pi_{1}\left(\mathbb{P}^{2}-\tilde{C}\right)$ is a central extension of $G$ by $\mathbb{Z} /(n+1) \mathbb{Z}$ :

$$
1 \rightarrow \mathbb{Z} /(n+1) \mathbb{Z} \rightarrow \tilde{G} \rightarrow G \rightarrow 1
$$

In particular, if $C$ is irreducible so is $\tilde{C}$ as well.

A natural question is which curves and fundamental groups can be obtained by this method. Also, one might ask if this method can be generalized, and what will be the effect of the general method on the relation between the fundamental groups of the original curve and the resulting curve.

In this paper we first generalize Uludağ's method to get new families of curves whose fundamental groups are controlled by the fundamental group of the original curve in the same manner. Precisely, instead of using only two fibers for performing the elementary transformations between Hirzebruch surfaces $F_{n}$, we allow any finite number of different fibers. Afterwards, we list properties of groups which are preserved by the methods. Also, we describe the curves obtained by the application of these methods to several types of plane curves. Then we present some infinite families of new Zariski pairs which can be obtained by the application of these methods.

Among the interesting results in this paper is the exploring of families of curves with deep singularities which yet have cyclic groups as the fundamental group 
of the complement (see the beginning of Section 4). Also, using the general construction, one can obtain more curves with finite and non-abelian fundamental groups and more Zariski pairs (see Section 6).

The paper is organized as follows. In Section 2 we present Uludağ's original construction. In Section 3, we present some generalizations of Uludağ's construction, and we prove that also in the general constructions, the obtained curve has a fundamental group which is a central extension of the original curve's fundamental group by a finite cyclic group. Section 4 deals with properties of groups which are preserved while applying the constructions. In Section 5, we describe precisely the families of plane curves which can be obtained by the general constructions and then we calculate the degrees of the new curves. At the end of this section, we describe some specific families of curves obtained by applying the constructions to several types of plane curves. In Section 6 we present some new examples of Zariski pairs obtained by these constructions.

\section{Uludă̆'s method}

The idea of the method is the following (it was partially introduced by ArtalBartolo [4] and Degtyarev [9], and the sequel was developed by Uludağ [32, 33]). If a curve $C_{2}$ is obtained from a curve $C_{1}$ by means of a Cremona transformation $\psi: \mathbb{P}^{2} \rightarrow \mathbb{P}^{2}$, then $\psi$ induces an isomorphism

$$
\mathbb{P}^{2}-\left(C_{2} \cup A\right) \cong \mathbb{P}^{2}-\left(C_{1} \cup B\right)
$$

where $A$ and $B$ are certain curve arrangements. Hence there is an induced isomorphism between their fundamental groups. The fundamental groups of the curves themselves are easy to compute by adding the relations which correspond to the arrangements.

Now, if we start with a curve $C_{1}$ whose fundamental group is known, one can find a curve $C_{2}$ whose fundamental group has not yet been known as being a fundamental group of a plane curve.

For these Cremona transformations one can use Hirzebruch surfaces $F_{n}, n \in$ $\mathbb{N} \cup\{0\}$. In principle, the Hirzebruch surfaces are $\mathbb{P}^{1}$-bundles over $\mathbb{P}^{1}$. It is known that two Hirzebruch surfaces can be distinguished by the self-intersection of their exceptional section (for $F_{n}$ the self-intersection of its exceptional section is $-n)$.

There are two types of elementary transformations, one transforms $F_{n}$ to $F_{n+1}$ for all positive $n$, and the other transforms $F_{n}$ to $F_{n-1}$ for all positive $n$ (these 
transformations are also called Nagata elementary transformations, see [18]). The first transformation blows up a point $O$ on the exceptional section, and then blows down the proper transform of the fiber passed through $O$. The second transformation blows up a point $O$ on one of the fibers $Q$, outside the exceptional section, and then blows down the proper transform of the fiber $Q$. The two transformations are schematically presented in Figures 1 and 2 respectively.
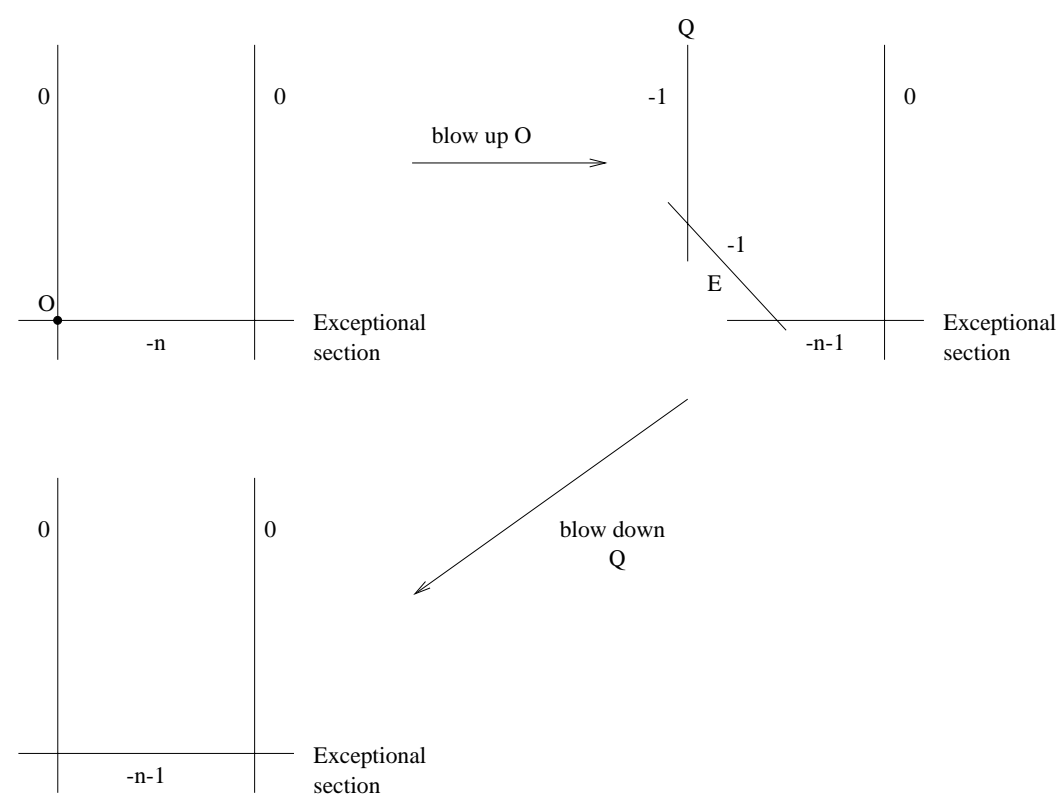

Figure 1: Elementary transformation from $F_{n}$ to $F_{n+1}$

Uludağ used a special type of Cremona transformations which can be described as follows. We start with a curve $C$ in $\mathbb{P}^{2}$, and an additional line $Q$ which intersects the curve transversally. We choose another line $P$ which also intersects the curve transversally, and meets $Q$ outside the curve $C$. Then we blow up the intersection point of the two lines. This yields the Hirzebruch surface $F_{1}$. Then we apply $n$ elementary transformations of the first type each time on the same fiber (which is the proper transform of the line $Q$ ) to reach $F_{n+1}$. Then we apply $n$ elementary transformations of the second type each time on the same fiber (which is the proper transform of the line $P$ ) to return back to $F_{1}$. Then we blow down the exceptional section (whose self-intersection is now -1 ), and we get again $\mathbb{P}^{2}$. This process defines a family of Cremona transformations from $\mathbb{P}^{2}$ to $\mathbb{P}^{2}$. 

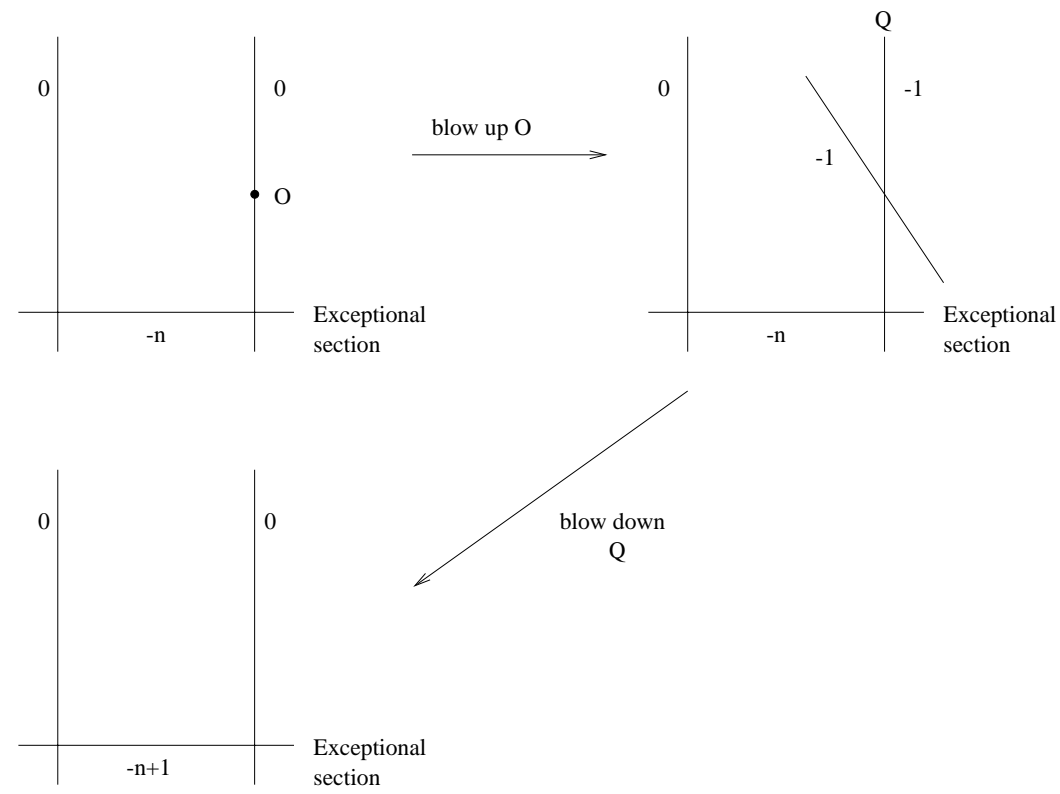

Figure 2: Elementary transformation from $F_{n}$ to $F_{n-1}$

For each $n$, we get a different Cremona transformation. Uludağ has shown in [32] that applying to a curve $C$ a Cremona transformation whose process reaches $F_{n+1}$, yields a new curve $\tilde{C}$ such that its fundamental group $\pi_{1}\left(\mathbb{P}^{2}-\tilde{C}\right)$ is a central extension of $\pi_{1}\left(\mathbb{P}^{2}-C\right)$ by a cyclic group of order $n+1$.

\section{Generalizations of Uludağ's method}

In this section we present some generalizations for Uludağ's method. These generalizations yield new ways to construct curves with deep singularities whose fundamental groups can be controlled, though they produce no more new groups as fundamental groups than the original method of Uludağ.

In the first step, we generalize Uludağ's method in the following way: instead of using the same fiber all the time to perform the elementary transformations of the first type, we will use an arbitrary finite number of different fibers for performing them (Subsection 3.3). In Subsection 3.4, we generalize our construction more, and we allow an arbitrary finite number of fibers for performing the elementary transformations of the second type too. In Subsection 3.5 we discuss a particularly interesting special case, where we perform all the transformations (of both types) on the same fiber. 
Before we pass to the generalizations of the methods and their proofs, we first have to introduce meridians and prove a lemma which will be needed in the sequel (Subsection 3.1). Also, we have to understand what happens to the fundamental group when we glue a line back to $\mathbb{P}^{2}$ (Subsection 3.2).

\subsection{Meridians and a generalization of Fujita's lemma}

As in Uludağ's proof [32], in order to find the relations induced by the additional lines, we have to calculate the meridians of these lines. We first recall the definition of a meridian of a curve $C$ at a point $p$ (see $[32,33])$ : Let $\Delta$ be a smooth analytical branch meeting $C$ transversally at $p$ and let $x_{0} \in \mathbb{P}^{2}-C$ be a base point. Take a path $\omega$ joining $x_{0}$ to a boundary point of $\Delta$, and define the meridian of $C$ at $p$ to be the loop $\mu_{p}=\omega \cdot \delta \cdot \omega^{-1}$, where $\delta$ is the boundary of $\Delta$, oriented in the positive sense (see Figure 3 ).

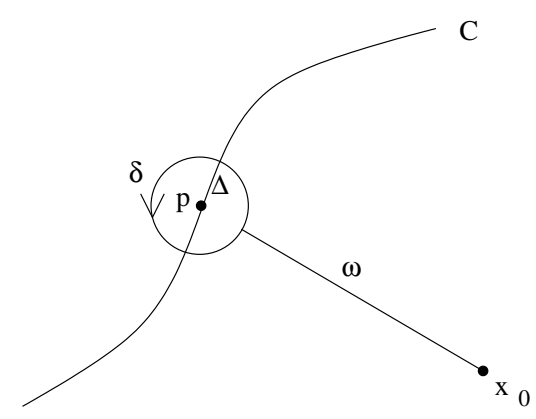

Figure 3: A meridian of a curve $C$ at a point $p$

For computing the meridians in our case, one has to use some rules. The first rule deals with the connection between the meridian of the curve $C$ at a point $p \in C$ before the blow up and the meridian of the exceptional section created by the blow up (see [32, p. 5]):

Claim 3.1 Let $\sigma_{p}: X \rightarrow \mathbb{P}^{2}$ be a blow up of the point $p \in C$, and let $E \subset X$ be the exceptional section. Let $C^{\prime}=\sigma_{p}^{-1}(C)$. Then, the loop $\sigma_{p}^{-1}\left(\mu_{p}\right)$ is the meridian of $E$ at a smooth point $q \in E-C^{\prime}$.

The second rule deals with the meridian at a nodal point:

Lemma 3.2 (Fujita [11, p. 540, Lemma 7.17]) Let $B$ be a ball centered at the origin $O$ of $\mathbb{C}^{2}$, and consider the curve $C$ defined by $x^{2}-y^{2}=0 . C$ has 
an ordinary double point at the origin and $\pi_{1}(B-C)=\mathbb{Z}^{2}$. Take meridians $\alpha$ and $\beta$ of $C$ on the branches $x=y$ and $x=-y$ at smooth points respectively. Then $\alpha \beta$ is a meridian of $C$ at the node $O$ (see Figure 4).

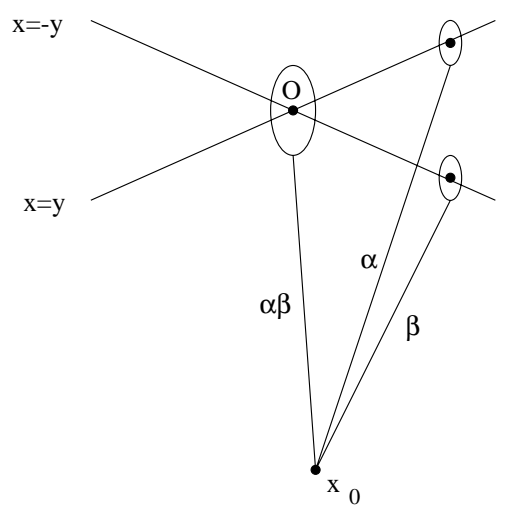

Figure 4: The situation of Fujita's lemma

For our generalizations, we need the following more general version of this lemma:

Lemma 3.3 Let $B$ be a ball centered at the origin $O$ of $\mathbb{C}^{2}$, and consider the curve $C$ defined by $\prod_{i=1}^{k}\left(y-m_{i} x\right)=0$ where the $m_{i}$ are some complex numbers, ordered by their distinct arguments, and $k \geq 3$. The curve $C$ has an ordinary singular point of multiplicity $k$ at the origin and $\pi_{1}(B-C)=\mathbb{Z} \oplus \mathbb{F}_{k-1}$, where $\mathbb{F}_{k-1}$ is the free group on $k-1$ generators. Take meridians $\alpha_{i}$ of $C$ on the branches $y=m_{i} x$ at smooth points respectively. Then $\alpha_{1} \alpha_{2} \cdots \alpha_{k}$ is a meridian of $C$ at the intersection point $O$.

Proof The fact that $\pi_{1}(B-C)=\mathbb{Z} \oplus \mathbb{F}_{k-1}$ is from [12] or [23]: Using van Kampen's method [34], the presentation of the group $\pi_{1}(B-C)$ is:

$$
\left\langle\alpha_{1}, \alpha_{2}, \cdots, \alpha_{k} \mid \alpha_{1} \alpha_{2} \cdots \alpha_{k}=\alpha_{2} \cdots \alpha_{k} \alpha_{1}=\cdots=\alpha_{k} \alpha_{1} \cdots \alpha_{k-1}\right\rangle,
$$

which can be written also as:

$$
\left\langle\alpha, \alpha_{2}, \cdots, \alpha_{k} \mid \alpha \alpha_{2}=\alpha_{2} \alpha, \alpha \alpha_{3}=\alpha_{3} \alpha, \cdots, \alpha \alpha_{k}=\alpha_{k} \alpha\right\rangle
$$

where $\alpha=\alpha_{1} \alpha_{2} \cdots \alpha_{k}$ (see [12]). This presentation can be written also as:

$$
\langle\alpha\rangle \oplus\left\langle\alpha_{2}, \cdots, \alpha_{k}\right\rangle \cong \mathbb{Z} \oplus \mathbb{F}_{k-1},
$$

and hence the generator of the cyclic group (which is also the center of the group) is $\alpha=\alpha_{1} \alpha_{2} \cdots \alpha_{k}$. 
In order to prove the lemma, we have to show that this generator is indeed the meridian of the exceptional section $E$ after we blow up the point $O$. When we blow the point $O$ up, we get Hirzebruch surface $F_{1} \rightarrow \mathbb{P}^{1}$. After deleting the exceptional section and another disjoint section (which corresponds to the line at infinity), we also throw away $k$ fibers corresponding to the $k$ lines which passed through $O$ before the blow up. The resulting affine surface can be decomposed as a product: $(\mathbb{C}-\{\mathrm{pt}\}) \times\left(\mathbb{P}^{1}-\{\mathrm{k}\right.$ points $\left.\}\right)$. Hence, its fundamental group can be decomposed into a direct sum too:

$$
\pi_{1}(\mathbb{C}-\{\mathrm{pt}\}) \oplus \pi_{1}\left(\mathbb{P}^{1}-\{\mathrm{k} \text { points }\}\right) \cong \mathbb{Z} \oplus \mathbb{F}_{k-1} .
$$

Now, since the cyclic group $\mathbb{Z}$ is in the center of the group $\mathbb{Z} \oplus \mathbb{F}_{k-1}$, its generator corresponds indeed to the meridian of the base $E$. Due to the fact that the generator of the cyclic group in the center is $\alpha=\alpha_{1} \alpha_{2} \cdots \alpha_{k}$, therefore since both the meridian and the generator $\alpha=\alpha_{1} \alpha_{2} \cdots \alpha_{k}$ generate the infinite cyclic group which is the center of the group, this generator is a meridian of the exceptional section $E$, and a meridian of the curve $C$ at the intersection point $O$ too.

\subsection{The effect on the fundamental group while gluing back a line}

In this short subsection, we prove a simple but useful lemma about the effect on the fundamental group when we glue a line back to $\mathbb{P}^{2}$.

Zaidenberg [35] has proved the following lemma:

Lemma 3.4 (Zaidenberg [35, Lemma 2.3(a)]) Let $D$ be a closed hypersurface in a complex manifold $M$. Then the group $\operatorname{Ker}\left\{i_{*}: \pi_{1}(M-D) \rightarrow \pi_{1}(M)\right\}$ is generated by the vanishing loops of $D$. In particular, if $D$ is irreducible, then, as a normal subgroup, this group is generated by any of these loops.

Let $C$ be a plane curve. Substituting $\mathbb{P}^{2}-C$ for $M$ and a line $L$ for $D$, we get that $\operatorname{Ker}\left\{i_{*}: \pi_{1}\left(\mathbb{P}^{2}-(C \cup L)\right) \rightarrow \pi_{1}\left(\mathbb{P}^{2}-C\right)\right\}$ is generated by the vanishing loops (=meridians) of $L$. Since $L$ is a line, we have:

$$
\operatorname{Ker}\left\{i_{*}: \pi_{1}\left(\mathbb{P}^{2}-(C \cup L)\right) \rightarrow \pi_{1}\left(\mathbb{P}^{2}-C\right)\right\}=\langle\langle\mu\rangle\rangle
$$

where $\mu$ is a meridian of $L$.

Therefore, it is easy to deduce the following lemma:

Lemma 3.5 Let $C$ be a plane projective curve and let $L$ be a line. Let $\mu$ be a meridian of $L$. Then:

$$
\pi_{1}\left(\mathbb{P}^{2}-(C \cup L)\right) /\langle\mu\rangle \cong \pi_{1}\left(\mathbb{P}^{2}-C\right)
$$




\subsection{The first generalization}

The first generalization of Uludağ's construction is as follows: Let $C$ be the initial plane curve, and let $n_{1}, \cdots, n_{k}$ be $k$ given natural numbers. In this construction, we have $k$ different lines $Q_{1}, \cdots, Q_{k}$ which all meet $C$ transversally. They intersect in a point $O$ outside $C$, in such a way that if we put a disk $D$ centered at $O$, the intersection points of the lines and the boundary of $D$ are organized counterclockwise on the boundary of $D$. The additional line $P$ passes via $O$ too and intersects $C$ transversally. Now we blow up the point $O$, in order to get Hirzebruch surface $F_{1}$. Then we apply $n_{i}$ elementary transformations of the first type on the proper transform of $Q_{i}$ for all $i=1, \ldots, k$. After this step, we have reached Hirzebruch surface $F_{\left(\sum_{i=1}^{k} n_{i}\right)+1}$. Now, we apply $\sum_{i=1}^{k} n_{i}$ elementary transformations of the second type on the proper transform of $P$. Then, we reach back $F_{1}$. At last, we blow down the exceptional section, and we get again $\mathbb{P}^{2}$. This defines a family of Cremona transformations from $\mathbb{P}^{2}$ to $\mathbb{P}^{2}$.

For any $k$-tuple $\left(n_{1}, \cdots, n_{k}\right) \in \mathbb{N}^{k}$, we get a different Cremona transformation, denoted by $T_{\left(n_{1}, \cdots, n_{k}\right)}$. We will show that the new curve $\tilde{C}=T_{\left(n_{1}, \cdots, n_{k}\right)}(C)$ has a fundamental group $\pi_{1}\left(\mathbb{P}^{2}-\tilde{C}\right)$ which is a central extension of $\pi_{1}\left(\mathbb{P}^{2}-C\right)$ by a finite cyclic group of order $\left(\sum_{i=1}^{k} n_{i}\right)+1$.

Remark 3.6 Before formulating the result, we note that the curve $\tilde{C}$ obtained by the transformation $T_{\left(n_{1}, \cdots, n_{k}\right)}$ can not be obtained by any successive applications of Uludağ's original method, since for instance two appropriate applications will yield an extension of order $n_{1}+n_{2}+2$ whereas the extension of the Cremona transformation $T_{\left(n_{1}, n_{2}\right)}\left(\right.$ for $k=2$ ) is of order $n_{1}+n_{2}+1$. Also the obtained singularities will be different (see Section 5).

Theorem 3.7 Let $C$ be a plane projective curve and $G=\pi_{1}\left(\mathbb{P}^{2}-C\right)$. Then for any $k$-tuple $\left(n_{1}, \cdots, n_{k}\right) \in \mathbb{N}^{k}$, the curve $\tilde{C}=T_{\left(n_{1}, \cdots, n_{k}\right)}(C)$ is birational to $C$, and its fundamental group $\tilde{G}=\pi_{1}\left(\mathbb{P}^{2}-\tilde{C}\right)$ is a central extension of $G$ by $\mathbb{Z} /\left(\left(\sum_{i=1}^{k} n_{i}\right)+1\right) \mathbb{Z}:$

$$
1 \rightarrow \mathbb{Z} /\left(\left(\sum_{i=1}^{k} n_{i}\right)+1\right) \mathbb{Z} \rightarrow \tilde{G} \rightarrow G \rightarrow 1
$$

Moreover, if $C$ has $r$ irreducible components so is $\tilde{C}$.

Proof We start with the observation that since the blow up and the blow down transformations are birational transformations and the fact that the Cremona 
transformation is composed of blow up and blow down transformations, we have that $\tilde{C}$ is birational to $C$, and the number of irreducible components is preserved by the construction.

Let $\tilde{P}=T_{\left(n_{1}, \cdots, n_{k}\right)}(P)$ and $\tilde{Q}_{i}=T_{\left(n_{1}, \cdots, n_{k}\right)}\left(Q_{i}\right)$ for $1 \leq i \leq k$ (one should notice here that $P$ is not actually mapped to $\tilde{P}$, since the transformation blows it down. We choose the new fiber that replaces the original one). This Cremona transformation defines an isomorphism:

$$
\mathbb{P}^{2}-\left(C \cup P \cup\left(\bigcup_{i=1}^{k} Q_{i}\right)\right) \cong \mathbb{P}^{2}-\left(\tilde{C} \cup \tilde{P} \cup\left(\bigcup_{i=1}^{k} \tilde{Q}_{i}\right)\right),
$$

which induces an isomorphism of the corresponding fundamental groups:

$$
\pi_{1}\left(\mathbb{P}^{2}-\left(C \cup P \cup\left(\bigcup_{i=1}^{k} Q_{i}\right)\right)\right) \cong \pi_{1}\left(\mathbb{P}^{2}-\left(\tilde{C} \cup \tilde{P} \cup\left(\bigcup_{i=1}^{k} \tilde{Q}_{i}\right)\right)\right) .
$$

In order to compute $\pi_{1}\left(\mathbb{P}^{2}-\tilde{C}\right)$, we have to add the relations correspond to gluing back the lines $\tilde{P}$ and $\tilde{Q}_{i}, 1 \leq i \leq k$.

Let $\beta$ and $\alpha_{i}$ be the meridians of the lines $P$ and $Q_{i}$ at smooth points respectively. Using Lemma 3.3, we get that the meridian of the curve $C \cup P \cup\left(\bigcup_{i=1}^{k} Q_{i}\right)$ at the point $O$ (which is the intersection of $k+1$ lines: $P, Q_{1}, \cdots, Q_{k}$ ) is $\beta \alpha_{1} \cdots \alpha_{k}$, and hence the meridian of $E$, which is the blow up of this point, is $\beta \alpha_{1} \cdots \alpha_{k}$ too.

We now compute the meridian of $\tilde{Q}_{i}(1 \leq i \leq k)$. In each elementary transformation of the first type on $Q_{i}$ and its proper transforms, we blow up the intersection point between the fiber and the exceptional section, and then we blow down the fiber. Hence, by Fujita's lemma (Lemma 3.2), each elementary transformation multiplies the current meridian of the proper transform of $Q_{i}$ by the meridian of the exceptional section. Therefore, we finally get that the meridian of $\tilde{Q}_{i}$ is $\left(\beta \alpha_{1} \cdots \alpha_{k}\right)^{n_{i}} \cdot \alpha_{i}$ (see Figure 5 for the effect of one elementary transformation of the first type where $k=2$ ). The elementary transformations on $Q_{i}$ and its proper transforms do not change the meridians of the other lines.

We have to compute the meridian of $\tilde{P}$ too. In each elementary transformation of the second type on $P$ and its proper transforms, we blow up a point on the fiber, and then we blow down the fiber. Hence, this type of elementary transformations preserves the current meridian and we get that the meridian of $\tilde{P}$ remains $\beta$ (see Figure 6 for the effect of one elementary transformation of the second type where $k=2$ ). The elementary transformations on $P$ and its proper transforms do not change the meridians of the other lines. 


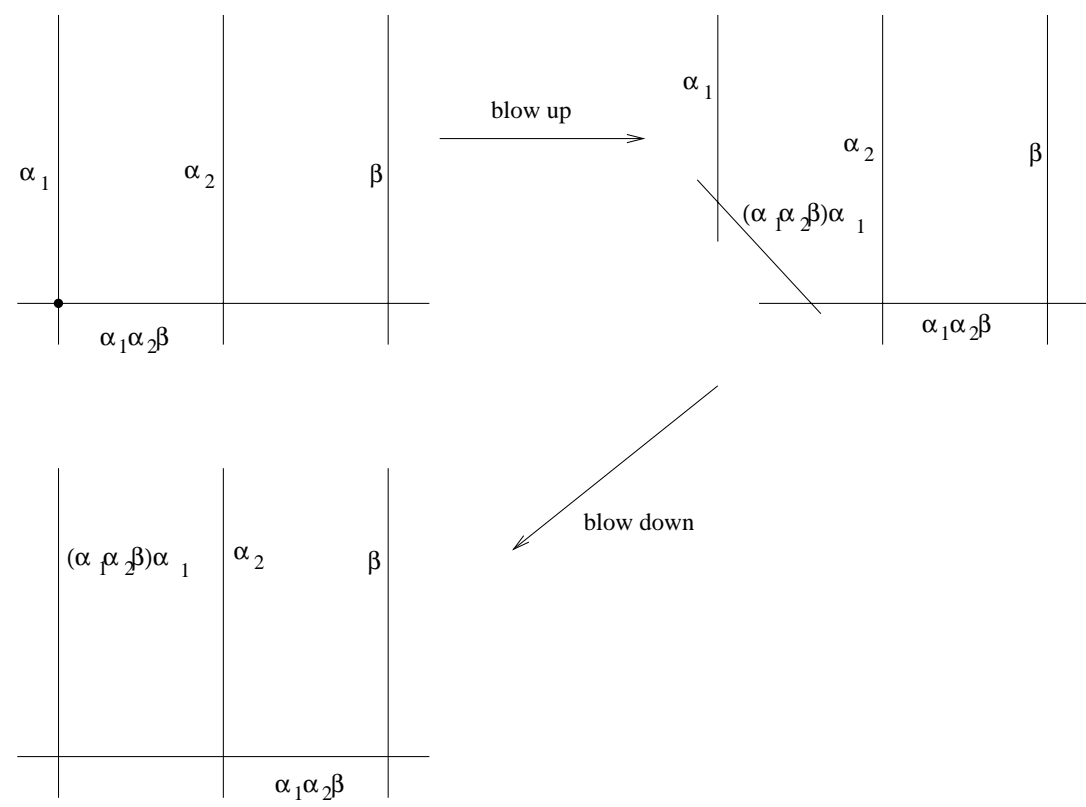

Figure 5: The effect of an elementary transformation of the first type on the meridians

Therefore, by Lemma 3.5, we conclude that:

$$
\begin{aligned}
& \pi_{1}\left(\mathbb{P}^{2}-\tilde{C}\right) \cong \\
& \quad \pi_{1}\left(\mathbb{P}^{2}-\left(\tilde{C} \cup \tilde{P} \cup\left(\bigcup_{i=1}^{k} \tilde{Q}_{i}\right)\right)\right) /\left\langle\beta,\left(\beta \alpha_{1} \cdots \alpha_{k}\right)^{n_{1}} \alpha_{1}, \cdots,\left(\beta \alpha_{1} \cdots \alpha_{k}\right)^{n_{k}} \alpha_{k}\right\rangle,
\end{aligned}
$$

which is equivalent to:

$\pi_{1}\left(\mathbb{P}^{2}-\tilde{C}\right) \cong \pi_{1}\left(\mathbb{P}^{2}-\left(\tilde{C} \cup \tilde{P} \cup\left(\bigcup_{i=1}^{k} \tilde{Q}_{i}\right)\right)\right) /\left\langle\beta,\left(\alpha_{1} \cdots \alpha_{k}\right)^{n_{1}} \alpha_{1}, \cdots,\left(\alpha_{1} \cdots \alpha_{k}\right)^{n_{k}} \alpha_{k}\right\rangle$

since the connecting relations between $\beta, \alpha_{1}, \cdots, \alpha_{k}$ are

$$
\beta \alpha_{1} \cdots \alpha_{k}=\alpha_{1} \cdots \alpha_{k} \beta=\cdots=\alpha_{k} \beta \alpha_{1} \cdots \alpha_{k-1}
$$

(see the proof of Lemma 3.3), because all the $k+1$ lines intersect in one point $O$.

Since

$$
\mathbb{P}^{2}-\left(C \cup P \cup\left(\bigcup_{i=1}^{k} Q_{i}\right)\right) \cong \mathbb{P}^{2}-\left(\tilde{C} \cup \tilde{P} \cup\left(\bigcup_{i=1}^{k} \tilde{Q}_{i}\right)\right),
$$

we can also write:

Algebraic 63 Geometric Topology, Volume 3 (2003) 

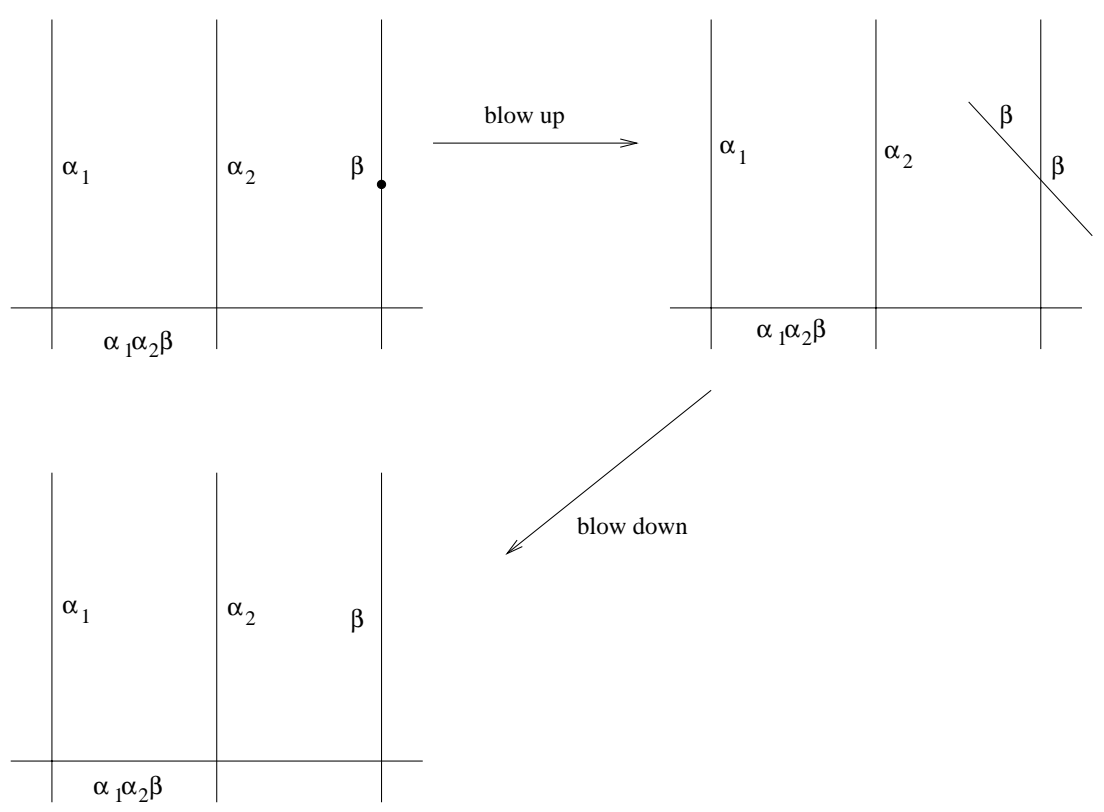

Figure 6: The effect of an elementary transformation of the second type on the meridians

$\pi_{1}\left(\mathbb{P}^{2}-\tilde{C}\right) \cong \pi_{1}\left(\mathbb{P}^{2}-\left(C \cup P \cup\left(\bigcup_{i=1}^{k} Q_{i}\right)\right)\right) /\left\langle\beta,\left(\alpha_{1} \cdots \alpha_{k}\right)^{n_{1}} \alpha_{1}, \cdots,\left(\alpha_{1} \cdots \alpha_{k}\right)^{n_{k}} \alpha_{k}\right\rangle$

When we pass to the quotient by $\langle\beta\rangle$ which corresponds to gluing the line $P$ back into $\mathbb{P}^{2}$ in the original configuration (by Lemma 3.5), we get that:

$$
\pi_{1}\left(\mathbb{P}^{2}-\tilde{C}\right) \cong \pi_{1}\left(\mathbb{P}^{2}-\left(C \cup\left(\bigcup_{i=1}^{k} Q_{i}\right)\right)\right) /\left\langle\left(\alpha_{1} \cdots \alpha_{k}\right)^{n_{1}} \alpha_{1}, \cdots,\left(\alpha_{1} \cdots \alpha_{k}\right)^{n_{k}} \alpha_{k}\right\rangle .
$$

While moving to this quotient, we get the following $k$ cyclic relations for the subgroup in the denominator:

$$
\alpha_{1} \alpha_{2} \cdots \alpha_{k}=\alpha_{2} \cdots \alpha_{k} \alpha_{1}=\cdots=\alpha_{k} \alpha_{1} \alpha_{2} \cdots \alpha_{k-1},
$$

since $\beta=1$ in the quotient. These relations can be presented also as the following set of relations, where $\alpha=\alpha_{1} \alpha_{2} \cdots \alpha_{k}$ :

$$
\left\{\alpha \alpha_{2}=\alpha_{2} \alpha, \alpha \alpha_{3}=\alpha_{3} \alpha, \cdots, \alpha \alpha_{k}=\alpha_{k} \alpha\right\}
$$

By Lemma 3.5, when we take the quotient of $\pi_{1}\left(\mathbb{P}^{2}-\left(C \cup\left(\bigcup_{i=1}^{k} Q_{i}\right)\right)\right)$ by the normal subgroup generated by the meridians of $Q_{1}, \cdots, Q_{k}$, we get $\pi_{1}\left(\mathbb{P}^{2}-C\right)$. 
Due to the fact that all the lines are intersected at $O$, then the subgroup generated by the meridians $Q_{1}, \cdots, Q_{k}$ is of the form (see the proof of Lemma $3.3)$ :

$$
\mathbb{Z} \oplus \mathbb{F}_{k-1} \cong\left\langle\alpha, \alpha_{2}, \cdots, \alpha_{k} \mid \alpha \alpha_{i}=\alpha_{i} \alpha, 2 \leq i \leq k\right\rangle
$$

where $\alpha=\alpha_{1} \cdots \alpha_{k}$.

We have

$$
\begin{gathered}
\frac{\pi_{1}\left(\mathbb{P}^{2}-\left(C \cup\left(\bigcup_{i=1}^{k} Q_{i}\right)\right)\right) /\left\langle\alpha^{n_{1}} \alpha_{1}, \cdots, \alpha^{n_{k}} \alpha_{k}\right\rangle}{\left\langle\alpha, \alpha_{2}, \cdots, \alpha_{k} \mid \alpha \alpha_{i}=\alpha_{i} \alpha, 2 \leq i \leq k\right\rangle /\left\langle\alpha^{n_{1}} \alpha_{1}, \cdots, \alpha^{n_{k}} \alpha_{k}\right\rangle} \cong \\
\cong \pi_{1}\left(\mathbb{P}^{2}-\left(C \cup\left(\bigcup_{i=1}^{k} Q_{i}\right)\right)\right) /\left\langle\alpha, \alpha_{2}, \cdots, \alpha_{k}\right\rangle
\end{gathered}
$$

where $\alpha=\alpha_{1} \cdots \alpha_{k}$, and therefore:

$$
\frac{\pi_{1}\left(\mathbb{P}^{2}-\tilde{C}\right)}{\left(\frac{\left\langle\alpha, \alpha_{2}, \cdots, \alpha_{k} \mid \alpha \alpha_{i}=\alpha_{i} \alpha, 2 \leq i \leq k\right\rangle}{\left\langle\alpha^{n_{1}} \alpha_{1}, \cdots, \alpha^{n} k \alpha_{k}\right\rangle}\right)} \cong \pi_{1}\left(\mathbb{P}^{2}-C\right)
$$

which can be written as the following extension:

$$
1 \rightarrow \frac{\left\langle\alpha, \alpha_{2}, \cdots, \alpha_{k} \mid \alpha \alpha_{i}=\alpha_{i} \alpha, 2 \leq i \leq k\right\rangle}{\left\langle\alpha^{n_{1}} \alpha_{1}, \cdots, \alpha^{n_{k}} \alpha_{k}\right\rangle} \rightarrow \pi_{1}\left(\mathbb{P}^{2}-\tilde{C}\right) \rightarrow \pi_{1}\left(\mathbb{P}^{2}-C\right) \rightarrow 1
$$

Hence, it remains to show that this extension is central and that

$$
\frac{\left\langle\alpha, \alpha_{2}, \cdots, \alpha_{k} \mid \alpha \alpha_{i}=\alpha_{i} \alpha, 2 \leq i \leq k\right\rangle}{\left\langle\alpha^{n_{1}} \alpha_{1}, \cdots, \alpha^{n_{k}} \alpha_{k}\right\rangle} \cong \mathbb{Z} /\left(\left(\sum_{i=1}^{k} n_{i}\right)+1\right) \mathbb{Z}
$$

The centrality of the extension is a little bit tricky. Although $\alpha_{i}$ does not commute with $\alpha_{j}(2 \leq i, j \leq k, i \neq j)$ in $\pi_{1}\left(P^{2}-\left(C \cup\left(\bigcup_{i=1}^{k} Q_{i}\right)\right)\right)$, the generators of the quotient group $\tilde{G}=\pi_{1}\left(\mathbb{P}^{2}-\tilde{C}\right)$ are the meridians of $C$ and only one more generator - $\alpha$ (the other generators $-\alpha_{2}, \cdots, \alpha_{k}$ - corresponding to the lines $Q_{2}, \cdots, Q_{k}$ in the bigger group $\pi_{1}\left(\mathbb{P}^{2}-\left(C \cup\left(\bigcup_{i=1}^{k} Q_{i}\right)\right)\right)$ disappear in $\tilde{G}$ by the additional relations $\left.\alpha^{n_{2}} \alpha_{2}, \cdots, \alpha^{n_{k}} \alpha_{k}\right)$. This generator indeed commutes with the meridians of $C$ in $\pi_{1}\left(\mathbb{P}^{2}-C\right.$ ) (as it is equal to the multiplication of all the $\alpha_{i}$ 's, which commute with the meridians of $C$ in $\pi_{1}\left(\mathbb{P}^{2}-C\right)$ since we choose the lines $Q_{1}, \cdots, Q_{k}$ to be all transversally intersected with $C$ ), and hence we get that the extension is central.

Now, we show that:

$$
\frac{\left\langle\alpha, \alpha_{2}, \cdots, \alpha_{k} \mid \alpha \alpha_{i}=\alpha_{i} \alpha, 2 \leq i \leq k\right\rangle}{\left\langle\alpha^{n_{1}} \alpha_{1}, \cdots, \alpha^{n_{k}} \alpha_{k}\right\rangle} \cong \mathbb{Z} /\left(\left(\sum_{i=1}^{k} n_{i}\right)+1\right) \mathbb{Z}
$$


Since

$$
\alpha^{n_{1}} \alpha_{1} \cdots \alpha^{n_{k}} \alpha_{k}=\alpha^{n_{1}+\cdots+n_{k}}\left(\alpha_{1} \cdots \alpha_{k}\right)=\alpha^{n_{1}+\cdots+n_{k}} \alpha=\alpha^{n_{1}+\cdots+n_{k}+1},
$$

we can change the presentation of the subgroup in the denominator to the following:

$$
\left\langle\alpha^{n_{1}+\cdots+n_{k}+1}, \alpha^{n_{2}} \alpha_{2}, \cdots, \alpha^{n_{k}} \alpha_{k} \mid \alpha \alpha_{i}=\alpha_{i} \alpha, 2 \leq i \leq k\right\rangle .
$$

On the other hand, one can see that the following is a presentation of $\mathbb{Z} \oplus \mathbb{F}_{k-1}$, which is the numerator:

$$
\mathbb{Z} \oplus \mathbb{F}_{k-1} \cong\left\langle\alpha, \alpha^{n_{2}} \alpha_{2}, \cdots, \alpha^{n_{k}} \alpha_{k} \mid \alpha \alpha_{i}=\alpha_{i} \alpha, 2 \leq i \leq k\right\rangle,
$$

since all the $\alpha_{i}, 2 \leq i \leq k$, can be achieved by the new set of generators. By these two new presentations, one can easily see that the quotient of these two groups is $\mathbb{Z} /\left(\left(\sum_{i=1}^{k} n_{i}\right)+1\right) \mathbb{Z}$ as needed, and hence Theorem 3.7 is proved.

\subsection{A slightly more general construction}

During the proof of the last theorem (before Figure 6), we have shown that elementary transformations of the second type do not affect the meridians of the fibers which we perform the transformations on. Therefore, we can generalize our construction to the following one: instead of performing all the elementary transformations of the second type on the same fiber $P$, we can apply them on several fibers $P_{1}, \cdots, P_{t}$, with the condition that the total number of applications of elementary transformations of the second type will be equal to the total number of applications of elementary transformations of the first type.

Using this observation, we can describe a slightly more general construction: Let $C$ be the initial plane curve, and let $n_{1}, \cdots, n_{k}$ and $m_{1}, \cdots, m_{l}$ be two sets of $k$ and $l$ given natural numbers, such that $\sum n_{i}=\sum m_{j}$. We start with $k+l$ different lines $Q_{1}, \cdots, Q_{k}$ and $P_{1}, \cdots, P_{l}$ which all meet $C$ transversally. They all intersect in a point $O$ outside $C$, in such a way that if we put a disk $D$ centered at $O$, the intersection points of the lines and the boundary of $D$ are organized counterclockwise on the boundary of $D$. Now we blow up the point $O$, in order to get Hirzebruch surface $F_{1}$. Then we apply $n_{i}$ elementary transformations of the first type on the proper transform of $Q_{i}$ for all $i=1, \ldots, k$. After this step, we have reached Hirzebruch surface $F_{\left(\sum_{i=1}^{k} n_{i}\right)+1}$. Now, we apply $m_{j}$ elementary transformations of the second type on the proper transform of $P_{j}$ for all $j=1, \ldots, l$. Since $\sum n_{i}=\sum m_{j}$, we reach back $F_{1}$. At last, we blow down the exceptional section (whose self-intersection is now -1 ), 
and we get again $\mathbb{P}^{2}$. This defines a family of Cremona transformations from $\mathbb{P}^{2}$ to $\mathbb{P}^{2}$.

For any $(k+l)$-tuple $\left(n_{1}, \cdots, n_{k}, m_{1}, \cdots, m_{l}\right) \in \mathbb{N}^{k+l}$ such that $\sum n_{i}=\sum m_{i}$, we get a Cremona transformation, denoted by $T_{\left(n_{1}, \cdots, n_{k} ; m_{1}, \cdots, m_{l}\right)}$. Then we can state:

Proposition 3.8 Let $C$ be a plane projective curve and $G=\pi_{1}\left(\mathbb{P}^{2}-C\right)$. Then for any $(k+l)$-tuple $\left(n_{1}, \cdots, n_{k}, m_{1}, \cdots, m_{l}\right) \in \mathbb{N}^{k+l}$ such that $\sum n_{i}=\sum m_{i}$, the curve $\tilde{C}=T_{\left(n_{1}, \cdots, n_{k} ; m_{1}, \cdots, m_{l}\right)}(C)$ is birational to $C$ and its fundamental group $\tilde{G}=\pi_{1}\left(\mathbb{P}^{2}-\tilde{C}\right)$ is a central extension of $G$ by $\mathbb{Z} /\left(\left(\sum_{i=1}^{k} n_{i}\right)+1\right) \mathbb{Z}$ :

$$
1 \rightarrow \mathbb{Z} /\left(\left(\sum_{i=1}^{k} n_{i}\right)+1\right) \mathbb{Z} \rightarrow \tilde{G} \rightarrow G \rightarrow 1
$$

Moreover, if $C$ has $r$ irreducible components so is $\tilde{C}$.

\subsection{An interesting special case}

Just before finishing the section of the constructions, we want to concentrate on an interesting special construction. In this construction, we perform all the elementary transformations from both types on the same fiber.

First, we define this construction precisely, and then we prove that the fundamental group of the resulting curve is again a central extension of the original curve, as we had in the previous constructions. We have to prove it, since the proof is slightly different from the proof of the previous case.

We start with a curve $C$ and one additional line $L$ in $\mathbb{P}^{2}$ which intersects $C$ transversally. Blow up a point $O$ on $L$ (which does not belong to $C$ ) in order to reach $F_{1}$. Then perform $n$ elementary transformations of the first type on the proper transform of $L$. Hence we reach $F_{n+1}$. Now, perform $n$ elementary transformations of the second type again on the proper transform of $L$. Now, blow down the exceptional section (which now has self-intersection -1) in order to return to $\mathbb{P}^{2}$. This construction defines a Cremona transformation from $\mathbb{P}^{2}$ to $\mathbb{P}^{2}$, which we denote by $T_{n}$. Then we have the following result:

Proposition 3.9 Let $C$ be a plane projective curve and $G=\pi_{1}\left(\mathbb{P}^{2}-C\right)$. Then for any natural number $n \in \mathbb{N}$, the curve $\tilde{C}=T_{n}(C)$ is birational to $C$ and its fundamental group $\tilde{G}=\pi_{1}\left(\mathbb{P}^{2}-\tilde{C}\right)$ is a central extension of $G$ by $\mathbb{Z} /(n+1) \mathbb{Z}$ :

$$
1 \rightarrow \mathbb{Z} /(n+1) \mathbb{Z} \rightarrow \tilde{G} \rightarrow G \rightarrow 1
$$

Moreover, if $C$ has $r$ irreducible components so is $\tilde{C}$. 
Proof As before, $\tilde{C}$ is birational to $C$, and number of irreducible components is preserved by $T_{n}$.

Let $\tilde{L}=T_{n}(L) . T_{n}$ induces an isomorphism of the corresponding fundamental groups:

$$
\pi_{1}\left(\mathbb{P}^{2}-(C \cup L)\right) \cong \pi_{1}\left(\mathbb{P}^{2}-(\tilde{C} \cup \tilde{L})\right)
$$

Let $\alpha$ be the meridian of $L$ at a smooth point. By Claim 3.1, we get that the meridian of the exceptional section $E$, which is the blow up of the point $O \in L$, is again $\alpha$.

After we apply the sequence of $n$ elementary transformations of the first type using the fiber $L$ and its proper transforms, we have by Fujita's lemma (Lemma 3.2) that the meridian of the proper transform of $L$ is $\alpha^{n+1}$. As before, the applications of elementary transformations of the second type and the final blow down do not change this meridian.

Therefore, by Lemma 3.5, we conclude that:

$$
\pi_{1}\left(\mathbb{P}^{2}-\tilde{C}\right) \cong \pi_{1}\left(\mathbb{P}^{2}-(\tilde{C} \cup \tilde{L})\right) /\left\langle\alpha^{n+1}\right\rangle
$$

As before, it is equivalent to:

$$
\pi_{1}\left(\mathbb{P}^{2}-\tilde{C}\right) \cong \pi_{1}\left(\mathbb{P}^{2}-(C \cup L)\right) /\left\langle\alpha^{n+1}\right\rangle .
$$

By Lemma $3.5, \pi_{1}\left(\mathbb{P}^{2}-(C \cup L)\right) /\langle\alpha\rangle=\pi_{1}\left(\mathbb{P}^{2}-C\right)$. Hence:

$$
\pi_{1}\left(\mathbb{P}^{2}-\tilde{C}\right) /\langle\alpha\rangle \cong \pi_{1}\left(\mathbb{P}^{2}-C\right),
$$

which can be written as the following extension:

$$
1 \rightarrow\left(\langle\alpha\rangle /\left\langle\alpha^{n+1}\right\rangle\right) \rightarrow \pi_{1}\left(\mathbb{P}^{2}-\tilde{C}\right) \rightarrow \pi_{1}\left(\mathbb{P}^{2}-C\right) \rightarrow 1 .
$$

Obviously, $\langle\alpha\rangle /\left\langle\alpha^{n+1}\right\rangle \cong \mathbb{Z} /(n+1) \mathbb{Z}$, and since $\alpha$ commutes with the generators of $\pi_{1}\left(\mathbb{P}^{2}-C\right)$ (since the line $L$ intersects $C$ transversally), the extension is central.

\section{Properties of groups preserved by the construc- tions}

In this section, we indicate some properties of the fundamental group which are preserved by the constructions of the previous section.

We start with an interesting property about the splitness of the central extension we have in the constructions. Using this property, we will show the 
following important property of the fundamental group of the resulting curve (Proposition 4.3): if we start with an irreducible curve which has a cyclic group as the fundamental group (such as smooth irreducible curves), then the resulting fundamental group will be cyclic too. The importance of this property is that although the constructions add to the curve deep singularities (as is proved in Proposition 5.2), the fundamental group of the curve is still cyclic. Hence, these constructions may yield families of plane curves which have some deep singularities but have cyclic fundamental groups.

Proposition 4.1 Let $C$ be a plane curve with $r$ irreducible components. Let $n \in \mathbb{N}$. Let $\tilde{C}$ be the curve whose fundamental group $\tilde{G}$ is obtained from $G=\pi_{1}\left(\mathbb{P}^{2}-C\right)$ by a central extension by $\mathbb{Z} /(n+1) \mathbb{Z}$. If the abelian group $H_{1}\left(\mathbb{P}^{2}-C\right)$ has $r$ direct summands and the orders of the summands are not coprime to $n+1$, then the extension does not split, i.e. $\tilde{G} ¥ G \oplus \mathbb{Z} /(n+1) \mathbb{Z}$.

Remark 4.2 In case of coprimeness, we obviously have

$$
\mathbb{Z} /(m n) \mathbb{Z} \cong \mathbb{Z} / m \mathbb{Z} \oplus \mathbb{Z} / n \mathbb{Z},
$$

and this is the reason for ruling this case out in the proposition.

Proof of Proposition 4.1 On the contrary, assume that $\tilde{G} \cong G \oplus \mathbb{Z} /(n+1) \mathbb{Z}$. As $H_{1}(X)$ is the abelinization of $\pi_{1}(X)$, we have that

$$
H_{1}\left(\mathbb{P}^{2}-\tilde{C}\right) \cong \mathrm{Ab}(\tilde{G}) \cong \mathrm{Ab}(G \oplus \mathbb{Z} /(n+1) \mathbb{Z}) \cong H_{1}\left(\mathbb{P}^{2}-C\right) \oplus \mathbb{Z} /(n+1) \mathbb{Z} .
$$

Since $n+1$ is not coprime to the orders of the summands of $H_{1}\left(\mathbb{P}^{2}-\tilde{C}\right)$, $H_{1}\left(\mathbb{P}^{2}-\tilde{C}\right)$ has $r+1$ proper direct summands. This contradicts the fact that $\tilde{C}$ has only $r$ irreducible components, as the number of irreducible components is preserved by the constructions.

Proposition 4.3 Let $C$ be an irreducible plane curve with a cyclic fundamental group $\mathbb{Z} / r \mathbb{Z}$. Let $n \in \mathbb{N}$. Let $\tilde{C}$ be the curve whose fundamental group $\tilde{G}$ is obtained from $G=\pi_{1}\left(\mathbb{P}^{2}-C\right)$ by a central extension by $\mathbb{Z} /(n+1) \mathbb{Z}$. Then $\tilde{G}=\pi_{1}\left(\mathbb{P}^{2}-\tilde{C}\right)$ is also cyclic of order $r(n+1)$.

Proof As $H_{1}(X)$ is the abelinization of $\pi_{1}(X)$, we have that $G=H_{1}\left(\mathbb{P}^{2}-C\right)$. Since $G$ is cyclic, $H_{1}\left(\mathbb{P}^{2}-C\right)$ has one direct summand, which equals the number of irreducible components in $C$ (one too). By the previous proposition, the extension does not split, and $H_{1}\left(\mathbb{P}^{2}-\tilde{C}\right)$ is cyclic too.

Since the extension is central, we have that $\tilde{G}$ is abelian of order $r(n+1)$. Hence, $\tilde{G}=H_{1}\left(\mathbb{P}^{2}-\tilde{C}\right)$. Therefore, $\tilde{G}$ is cyclic of order $r(n+1)$. 
Remark 4.4 The condition that the curve is irreducible is essential, since if we take a reducible curve with a cyclic fundamental group, it is not guaranteed that the resulting curve will have a cyclic fundamental group. For example, if we start with a curve $C$ consists of two intersecting lines whose fundamental group $\pi_{1}\left(\mathbb{P}^{2}-C\right)=\mathbb{Z}$ is cyclic, and we apply on it Uludağ's method for $n=1$, we get that the resulting curve $\tilde{C}$ has a fundamental group $\pi_{1}\left(\mathbb{P}^{2}-\tilde{C}\right)=\mathbb{Z} \oplus(\mathbb{Z} / 2 \mathbb{Z})$ (see after Proposition 5.11) which is not cyclic.

Let $p$ be a prime number. Then:

Remark 4.5 Let $C$ be a plane curve with a fundamental group $G$ which is a $p$-group. Let $n \in \mathbb{N}$. Let $\tilde{C}$ be the curve whose fundamental group $\tilde{G}$ is obtained from $G=\pi_{1}\left(\mathbb{P}^{2}-C\right)$ by a central extension by $\mathbb{Z} /(n+1) \mathbb{Z}$. Then: if $n+1=p^{l}$ for some $l$, then $\tilde{G}=\pi_{1}\left(\mathbb{P}^{2}-\tilde{C}\right)$ is also a $p$-group.

Proof From the extension, we get that $\tilde{G} /(\mathbb{Z} /(n+1) \mathbb{Z}) \cong G$. Since $n+1=p^{l}$, $\mathbb{Z} /(n+1) \mathbb{Z}$ is a $p$-group, and since $G$ is also a $p$-group, then $\tilde{G}$ is a $p$-group too.

Remark 4.6 Let $C$ be a plane curve with a finite fundamental group $G$. Let $n \in \mathbb{N}$. Let $\tilde{C}$ be the curve whose fundamental group $\tilde{G}$ is obtained from $G=\pi_{1}\left(\mathbb{P}^{2}-C\right)$ by a central extension by $\mathbb{Z} /(n+1) \mathbb{Z}$. If $(|G|, n+1)=1$, then the fundamental group $\tilde{G}$ of the resulting curve is a direct sum of $G$ and $\mathbb{Z} /(n+1) \mathbb{Z}:$

$$
\tilde{G} \cong G \oplus \mathbb{Z} /(n+1) \mathbb{Z}
$$

Proof Use Theorem 7.77 of [24] that "if $Q$ is a finite group, $K$ is a finite abelian group, and $(|K|,|Q|)=1$, then an extension $G$ of $Q$ by $K$ is a semidirect product of $K$ and $Q$ ", and since the extension is central, semidirect products become direct products.

In the following proposition, we list some more properties of the fundamental group which are preserved by the constructions. Before stating it, we remind some definitions. A group is called polycyclic if it has a subnormal series with cyclic factors. A group is called supersolvable if it has a normal series with cyclic factors. We say that a group $G$ is nilpotent if its lower central series reaches 1 (see for example [24]). 
Proposition 4.7 Let $C$ be a plane curve with a fundamental group $G$. Let $n \in \mathbb{N}$. Let $\tilde{C}$ be the curve whose fundamental group $\tilde{G}$ is obtained from $G=\pi_{1}\left(\mathbb{P}^{2}-C\right)$ by a central extension by $\mathbb{Z} /(n+1) \mathbb{Z}$. Then if $G$ has one of the following properties, the fundamental group $\tilde{G}$ of the resulting curve has that property too:

(1) Finite.

(2) Non-abelian.

(3) Solvable.

(4) Supersolvable.

(5) Polycyclic.

(6) Nilpotent.

Proof (1-2) Trivial.

(3) From the extension, we get that $\tilde{G} /(\mathbb{Z} /(n+1) \mathbb{Z}) \cong G$. But it is known $[24$, Theorem 5.17] that if $\mathbb{Z} /(n+1) \mathbb{Z}$ and $G$ are both solvable, then $\tilde{G}$ is solvable too.

(4) From the extension, we have that $\tilde{G} /(\mathbb{Z} /(n+1) \mathbb{Z}) \cong G$. It is easy to show (very similar to the solvable case, see [24, Theorem 5.17]) that if $\mathbb{Z} /(n+1) \mathbb{Z}$ and $G$ are both supersolvable, then $\tilde{G}$ is supersolvable too.

(5) Same proof as (4).

(6) Since $\tilde{G}$ is a central extension of $G$ by $\mathbb{Z} /(n+1) \mathbb{Z}$ for a given $n$, we get that $\tilde{G} /(\mathbb{Z} /(n+1) \mathbb{Z}) \cong G$ where $\mathbb{Z} /(n+1) \mathbb{Z} \leq Z(\tilde{G})$. But it is easy to see that if $\mathbb{Z} /(n+1) \mathbb{Z} \leq Z(\tilde{G})$ and $G$ is nilpotent, then $\tilde{G}$ is nilpotent too (see for example [24, p. 117, Exercise 5.38]).

We note here that if $G$ is a nilpotent group of class $c$ (means that the last nonzero term of the lower central series is the $c$-th term), then $\tilde{G}$ is a nilpotent group of class $c$ or $c+1$. It will be of class $c$ if and only if the 2-cocycle defining the extension is symmetric (see [25]).

Here we indicate one more family of group properties which are preserved by the constructions.

Remark 4.8 Let $C$ be a plane curve with a fundamental group $G$ which has a subgroup $N$ of finite index with a special property (for example: solvable, nilpotent, etc.). Then the fundamental group $\tilde{G}$ of the resulting curve $\tilde{C}$ has a subgroup $\tilde{N}$ of finite index with the same special property too.

In particular, if $G$ is virtually-nilpotent or virtually-solvable, then $\tilde{G}$ has the same property as well. 
Proof Let $n \in \mathbb{N}$. Since $\tilde{G}$ is an extension of $G$ by $\mathbb{Z} /(n+1) \mathbb{Z}$, then $\tilde{G} /(\mathbb{Z} /(n+1) \mathbb{Z}) \cong G$. Hence there exists $\tilde{N} \leq \tilde{G}$ such that $\tilde{N} /(\mathbb{Z} /(n+1) \mathbb{Z}) \cong$ $N$. Obviously $[\tilde{G}: \tilde{N}]=[G: N]<\infty$. Since $\tilde{G}$ is a central extension, one can show that also $\tilde{N}$ is a central extension of $N$ by $\mathbb{Z} /(n+1) \mathbb{Z}$ (since $\mathbb{Z} /(n+1) \mathbb{Z}$ is a subgroup of the intersection of $Z(\tilde{G})$ and $\tilde{N}$, and hence in $Z(\tilde{N}))$. Therefore, one can apply Proposition 4.7 to show that the properties of $N$ are moved to $\tilde{N}$.

Remark 4.9 Note that all the results of this section hold also for the constructions of Oka [20] and Shimada [26], since also in their constructions the fundamental group of the resulting curve is a central extension of the fundamental group of the original curve by a cyclic group.

\section{The curves obtained by the constructions}

In this section we investigate the curves which can be obtained using Uludağ's original construction and the general constructions we have presented in the previous sections. In the first subsection, we will describe the types of singularities which are added by the constructions. In Subsection 5.2 we compute the degrees of the resulting curves. In the next subsections, we describe some families of curves which can be obtained by applying the constructions on several different types of curves.

\subsection{The types of singularities which are added to the curves}

At the beginning of this subsection, we want to fix a notation for singular points. We follow the notations of Flenner and Zaidenberg [10]. Although in general it is possible that a singular point splits into several points by a blow up, in most of our cases (except for Proposition 3.8), only one singular point appears by a blow up. Hence, any singular point $P$ has a very simple resolution by a sequence of $s$ blow ups. We denote by $t_{i}>1(1 \leq i \leq s)$ the multiplicity of the curve at $P$ before the $i$-th blow up. Then $\left[t_{1}, \cdots, t_{s}\right]$ is called the type of the singularity. If we have a sequence of $r$ equal multiplicities $l$, we abbreviate it by $l_{r}$. For example, $[2,2,2]=\left[2_{3}\right]$ corresponds to a ramphoid cusp, which has to be blown up three times (each time of multiplicity 2) for smoothing it.

To simplify the description, we also introduce the notion of a $d$-tacnode. 
Definition 5.1 A $d$-tacnode is a singular point where $d$ smooth branches of the curve have a common tangent at the same point. A $d$-tacnode of order $k$ is a $d$-tacnode such that the higher derivatives of the branches are also equal up to order $k$.

For example, the usual tacnode is 2 -tacnode of order 1 .

Here we describe the singularities which are added to the curve during these constructions:

Proposition 5.2 Let $C$ be a curve of degree $d$. Let $\left(n_{1}, \cdots, n_{k}\right) \in \mathbb{N}^{k}$ be a $k$-tuple. Let $\tilde{C}=T_{\left(n_{1}, \cdots, n_{k}\right)}(C)$ (see Theorem 3.7).

Then $\tilde{C}$ has $k+1$ additional singularities to those of $C: k d$-tacnodes of order $n_{i}-1(1 \leq i \leq k)$, and another singular point which is a blow down of a $d$-tacnode of order $n_{1}+\cdots+n_{k}-1$ (i.e., the curve has the following additional singularities: $\left[d_{n_{1}}\right], \cdots,\left[d_{n_{k}}\right]$ and $\left.\left[d\left(n_{1}+\cdots+n_{k}\right), d_{\left(n_{1}+\cdots+n_{k}\right)}\right]\right)$.

Proof The first blow-up (from $\mathbb{P}^{2}$ to $F_{1}$ ) does not change the curve. Each sequence of $n_{i}$ elementary transformations of the first type on the fiber $Q_{i}$ and its proper transforms creates one $d$-tacnode of order $n_{i}-1$ : The first elementary transformation of the first type creates one intersection point (of $d$ branches). The second elementary transformation of the first type converts it into a $d$-tacnode of order 1 , and another $n_{i}-2$ elementary transformations of the first type convert it into a $d$-tacnode of order $n_{i}-1$. This $d$-tacnode is located at the proper transform of $Q_{i}$, outside the exceptional section.

The sequence of $n_{1}+\cdots+n_{k}$ elementary transformations of the second type (from $F_{n_{1}+\cdots+n_{k}+1}$ to $F_{1}$ ) creates another $d$-tacnode of order $n_{1}+\cdots+n_{k}-1$, as in the case of the elementary transformations of the first type. The difference is that now this $d$-tacnode is located at the exceptional section. Hence, when we blow down this section in order to return to $\mathbb{P}^{2}$, this $d$-tacnode of order $n_{1}+\cdots+n_{k}-1$ is blown down too to a more complicated singular point.

The following remark states the situation after applying only Uludağ's original construction.

Remark 5.3 Let $C$ be a curve of degree $d$. Let $n \in \mathbb{N}$. Let $\tilde{C}$ be the curve obtained by Uludağ's original construction for this $n$ (see Section 2).

Then $\tilde{C}$ has two additional singularities to those of $C$ : a $d$-tacnode of order $n-1$, and another singular point which is a blow down of a $d$-tacnode of order $n-1$ (i.e., the curve has the following additional singularities: $\left[d_{n}\right]$ and $\left.\left[d n, d_{n}\right]\right)$. 
Here we describe the situation concerning the special case (Proposition 3.9).

Proposition 5.4 Let $C$ be a curve of degree $d$. Let $n \in \mathbb{N}$. Let $\tilde{C}=T_{n}(C)$.

Then $\tilde{C}$ has one additional singularity to those of $C$ : a blow down of a $d$ tacnode of order $2 n-1$ (i.e., the curve has the following additional singularity: $\left.\left[2 n d, d_{2 n}\right]\right)$.

Proof As before, the first blow-up does not change the curve. The sequence of $n$ elementary transformations of the first type creates one $d$-tacnode of order $n-1$. Since we apply the second sequence of $n$ elementary transformations of the second type on the same fiber, we continue to deepen this singularity into a $d$-tacnode of order $2 n-1$ which is now located also at the exceptional section. Hence, when we blow down this section in order to return to $\mathbb{P}^{2}$, this $d$-tacnode of order $2 n-1$ is blown down too to a more complicated singular point.

The description of the curves obtained by Proposition 3.8 is a little bit more complicated: In this case we indeed have a singular point which is splitted after the first blow-up into several singular points.

Proposition 5.5 Let $C$ be a curve of degree d. Let $\left(n_{1}, \cdots, n_{k}, m_{1}, \cdots, m_{l}\right) \in$ $\mathbb{N}^{k+l}$ be a $(k+l)$-tuple such that $\sum n_{i}=\sum m_{j}$. Let $\tilde{C}=T_{\left(n_{1}, \cdots, n_{k} ; m_{1}, \cdots, m_{l}\right)}(C)$ (see Proposition 3.8).

Then $\tilde{C}$ has $k+1$ additional singularities to those of $C: k d$-tacnodes of order $n_{i}-1 \quad(1 \leq i \leq k)$, and another singular point which is a blow down of $l$ $d$-tacnodes of order $m_{j}-1(1 \leq j \leq l)$ on the exceptional section of that blow down (i.e., the curve has the following additional singularities: $\left[d_{n_{1}}\right], \cdots,\left[d_{n_{k}}\right]$ and $\left.\left[d\left(n_{1}+\cdots+n_{k}\right),\left(\left[d_{m_{1}}\right], \cdots,\left[d_{m_{l}}\right]\right)\right]\right)$.

Proof The proof is similar to the proofs of the previous propositions. As before, the elementary transformations of the first type create $k d$-tacnodes of order $n_{i}-1$.

The sequences of $m_{j}(1 \leq j \leq l)$ elementary transformations of the second type on the fibers $P_{1}, \cdots, P_{l}$ create $l d$-tacnodes of order $m_{j}-1$, which are all located at the exceptional section. Hence, when we blow this section down in order to return to $\mathbb{P}^{2}$, these $l d$-tacnodes are blown down together to a complicated singular point. 


\subsection{Change of the degree of the curve}

In this subsection, we compute the degree of the resulting curve:

Proposition 5.6 Let $C$ be a plane projective curve of degree $d$. Let $\left(n_{1}, \cdots, n_{k}\right) \in \mathbb{N}^{k}$. Let $\tilde{C}=T_{\left(n_{1}, \cdots, n_{k}\right)}(C)$ (see Theorem 3.7).

Then the degree of the resulting curve is $d\left(n_{1}+\cdots+n_{k}+1\right)$.

Proof Let $\tilde{d}$ be the degree of $\tilde{C}$. We have to show that $\tilde{d}=d\left(n_{1}+\cdots+n_{k}+1\right)$. When we blow up once one of the singularities, say $P$, in order to resolve it, we have to decrease the self-intersection of the original curve $\tilde{C}$ by $\left(\operatorname{mult}_{\tilde{C}} P\right)^{2}$ (where mult $\tilde{C} P$ is the local multiplicity of $\tilde{C}$ at $P$ ) to get the self-intersection of the curve $\tilde{C}$ after the blow-up. Since this is the data which is given by the types of the singularities, one can compute easily the change in the self-intersection.

So, we start with $\tilde{C}$ whose self-intersection is $\tilde{d}^{2}$, since $\tilde{C}$ is in $\mathbb{P}^{2}$. For all $1 \leq i \leq k$, the $n_{i}$ blow-ups of the singular point $\left[d_{n_{i}}\right]$ yield a decreasing of the self-intersection by $n_{i} \cdot d^{2}$, since the multiplicity of the curve at the singular point is $d$. The $n_{1}+\cdots+n_{k}+1$ blow-ups of the singular point of the type $\left[d\left(n_{1}+\cdots+n_{k}\right), d_{n_{1}+\cdots+n_{k}}\right]$ yield an additional decreasing of the self-intersection by $\left(d\left(n_{1}+\cdots+n_{k}\right)\right)^{2}+\left(n_{1}+\cdots+n_{k}\right) d^{2}$, since the multiplicity of the curve at the singular point in the first blow up is $d\left(n_{1}+\cdots+n_{k}\right)$ and in the other blow ups it is again $d$. After all these blow-ups, we reach the original curve $C$ in $\mathbb{P}^{2}$ and hence its self-intersection is $d^{2}$. Therefore, we have the following equation:

$$
\tilde{d}^{2}-\sum_{i=1}^{k}\left(n_{i} \cdot d^{2}\right)-\left(\left(d\left(n_{1}+\cdots+n_{k}\right)\right)^{2}+\left(n_{1}+\cdots+n_{k}\right) d^{2}\right)=d^{2}
$$

and hence $\tilde{d}^{2}=d^{2}\left(n_{1}+\cdots+n_{k}+1\right)^{2}$, which gives us $\tilde{d}=d\left(n_{1}+\cdots+n_{k}+1\right)$ as needed.

One can perform the same computations also for the curves obtained by the constructions presented in Propositions 3.8 and 3.9 (see Propositions 5.5 and 5.4 respectively for the descriptions of the additional singularities). Hence, Proposition 5.6 holds for those curves too.

\subsection{Families of curves obtained by starting with smooth irre- ducible curves}

In this subsection we describe the families of curves which are obtained by Uludağ's original construction and its generalizations if we apply them to a 
smooth irreducible curve $C$ of degree $d$ (and therefore $\pi_{1}\left(\mathbb{P}^{2}-C\right) \cong \mathbb{Z} / d \mathbb{Z}$, see Zariski [36]).

Proposition 5.7 Let $C$ be a smooth irreducible curve of degree $d$. Let $n \in \mathbb{N}$. Let $\tilde{C}$ be the curve obtained by Uludağ's construction in such a way that its fundamental group is a central extension of $G=\pi_{1}\left(\mathbb{P}^{2}-C\right)=\mathbb{Z} / d \mathbb{Z}$ by $\mathbb{Z} /(n+1) \mathbb{Z}$

Then for $n=1, \tilde{C}$ has an intersection point of $d$ smooth branches and one $d$-tacnode (i.e., the curve has the singularities: $[d]$ and $\left[d_{2}\right]$ ).

For $n \geq 2, \tilde{C}$ has a $d$-tacnode of order $n-1$, and another singular point which is a blow down of a $d$-tacnode of order $n-1$ (i.e., the curve has the following singularities: $\left[d_{n}\right]$ and $\left.\left[d n, d_{n}\right]\right)$.

The degree of the resulting curve is $d(n+1)$.

Proof Since a smooth curve has no singularities, then the only singularities of the resulting curve are those which were created by Uludağ's construction (see Remark 5.3). Therefore the curve has only the singularities described in Remark 5.3.

The degree of the curve can be computed directly by Proposition 5.6.

For the particular case $d=2$ and $n=1$, we indeed get a quadric with a node and a tacnode, and its equation can be found in [19, p. 147, case 2]: $\left(x^{2}+y^{2}-3 x\right)^{2}=4 x^{2}(2-x)$.

Using Proposition 4.3, we have that

$$
\pi_{1}\left(\mathbb{P}^{2}-\tilde{C}\right) \cong \mathbb{Z} /(d(n+1)) \mathbb{Z}
$$

Now, we describe the family of curves which are obtained by the general construction (Subsection 3.3).

Proposition 5.8 Let $C$ be a smooth irreducible curve. Let $\left(n_{1}, \cdots, n_{k}\right) \in \mathbb{N}^{k}$ be a $k$-tuple. Let $\tilde{C}=T_{\left(n_{1}, \cdots, n_{k}\right)}(C)$ (see Theorem 3.7).

For every $1 \leq i \leq k, \tilde{C}$ has a $d$-tacnode of order $n_{i}-1$, and another singular point which is a blow down of a $d$-tacnode of order $n_{1}+\cdots+n_{k}-1$ (i.e., the curve has the following singularities: $\left[d_{n_{1}}\right], \cdots,\left[d_{n_{k}}\right]$ and $\left.\left[d\left(n_{1}+\cdots+n_{k}\right), d_{n_{1}+\cdots+n_{k}}\right]\right)$. The degree of the resulting curve is $d\left(n_{1}+\cdots+n_{k}+1\right)$. 
Proof Similar to the proof of the previous proposition, but here we use the results of Proposition 5.2.

Using Proposition 4.3 again, we have that

$$
\pi_{1}\left(\mathbb{P}^{2}-\tilde{C}\right) \cong \mathbb{Z} /\left(d\left(n_{1}+\cdots+n_{k}+1\right)\right) \mathbb{Z} .
$$

\subsection{Families of curves obtained by starting with line arrange- ments}

In this subsection we describe the families of curves and their groups which are obtained by Uludağ's original construction and its generalizations if we apply them to some types of line arrangements.

Proposition 5.9 Let $\mathcal{L}$ be a line arrangement consisting of $m$ lines intersecting in one point. Let $n \in \mathbb{N}$. Let $\tilde{\mathcal{L}}$ be the curve obtained by Uludağ's construction in such a way that its fundamental group is a central extension of $G=\pi_{1}\left(\mathbb{P}^{2}-\mathcal{L}\right)=\mathbb{F}_{m-1}$ by $\mathbb{Z} /(n+1) \mathbb{Z}$.

Then for $n=1, \tilde{\mathcal{L}}$ has two intersection points of $m$ smooth branches and one $m$-tacnode (i.e., the curve has the following singularities: $[m],[m]$ and $\left[m_{2}\right]$ ).

For $n \geq 2, \tilde{\mathcal{L}}$ has one intersection points of $m$ smooth branches, one $m$-tacnode of order $n-1$, and another singular point which is a blow down of a $m$-tacnode of order $n-1$ (i.e., the curve has the following singularities: $[m],\left[m_{n}\right]$ and $\left.\left[m n, m_{n}\right]\right)$.

The degree of the resulting curve is $m(n+1)$.

Proof Since $\mathcal{L}$ has one intersection point of $m$ smooth branches, then the singularities of the resulting curve are those which were created by Uludağ's construction (see Remark 5.3) and an additional singularity which was in $\mathcal{L}$.

The degree of the curve is computed directly by Proposition 5.6.

Since $H^{2}\left(\mathbb{F}_{m-1}, \mathbb{Z} /(n+1) \mathbb{Z}\right)$ is trivial, then we get that:

$$
\pi_{1}\left(\mathbb{P}^{2}-\tilde{\mathcal{L}}\right) \cong \mathbb{F}_{m-1} \oplus \mathbb{Z} /(n+1) \mathbb{Z} .
$$

Now, we describe the family of curves which are obtained by the general construction (Subsection 3.3). 
Proposition 5.10 Let $\mathcal{L}$ be a line arrangement consisting of $m$ lines intersecting in one point. Let $\left(n_{1}, \cdots, n_{k}\right) \in \mathbb{N}^{k}$ be a $k$-tuple. Let $\tilde{\mathcal{L}}=T_{\left(n_{1}, \cdots, n_{k}\right)}(\mathcal{L})$ (see Theorem 3.7).

Then: in addition to the original intersection point of $\mathcal{L}$, for every $1 \leq i \leq k$, $\tilde{\mathcal{L}}$ has a $m$-tacnode of order $n_{i}-1$, and another singular point which is a blow down of a $m$-tacnode of order $n_{1}+\cdots+n_{k}-1$ (i.e., the curve has the following singularities: $[m],\left[m_{n_{1}}\right], \cdots,\left[m_{n_{k}}\right]$ and $\left.\left[m\left(n_{1}+\cdots+n_{k}\right), m_{n_{1}+\cdots+n_{k}}\right]\right)$.

The degree of the resulting curve is $m\left(n_{1}+\cdots+n_{k}+1\right)$.

Proof Similar to the proof of the previous proposition, but here we use the results of Proposition 5.2.

As before, since $H^{2}\left(\mathbb{F}_{m-1}, \mathbb{Z} /\left(n_{1}+\cdots+n_{k}+1\right) \mathbb{Z}\right)$ is trivial, then we get that:

$$
\pi_{1}\left(\mathbb{P}^{2}-\tilde{\mathcal{L}}\right) \cong \mathbb{F}_{m-1} \oplus \mathbb{Z} /\left(n_{1}+\cdots+n_{k}+1\right) \mathbb{Z} .
$$

Now we deal with another important type of line arrangements: lines in a general position, which means that there is no intersection of more than two lines in a point. We describe the family of curves which are obtained by the general construction (Subsection 3.3).

Proposition 5.11 Let $\mathcal{L}$ be a line arrangement consisting of $m$ lines in a general position. Let $\left(n_{1}, \cdots, n_{k}\right) \in \mathbb{N}^{k}$ be a $k$-tuple. Let $\tilde{\mathcal{L}}=T_{\left(n_{1}, \cdots, n_{k}\right)}(\mathcal{L})$ (see Theorem 3.7).

Then: in addition to the $\left(\begin{array}{c}m \\ 2\end{array}\right)$ nodal points of $\mathcal{L}$, for every $1 \leq i \leq k, \tilde{\mathcal{L}}$ has a $m$-tacnode of order $n_{i}-1$, and another singular point which is a blow down of a $m$-tacnode of order $n_{1}+\cdots+n_{k}-1$ (i.e., the curve has the following singularities: $\left[m_{n_{1}}\right], \cdots,\left[m_{n_{k}}\right],\left[m\left(n_{1}+\cdots+n_{k}\right), m_{n_{1}+\cdots+n_{k}}\right]$ and $\left(\begin{array}{c}m \\ 2\end{array}\right)$ singularities of the type $[2])$.

The degree of the resulting curve is $m\left(n_{1}+\cdots+n_{k}+1\right)$.

Proof Since $\mathcal{L}$ has $\left(\begin{array}{c}m \\ 2\end{array}\right)$ nodal points, then the singularities of the resulting curve are those which were created by the general construction (see Proposition $5.2)$ and $\left(\begin{array}{c}m \\ 2\end{array}\right)$ nodal points.

The degree of the curve is computed directly by Proposition 5.6. 
Since a central extension of $\mathbb{Z}^{m-1}$ by $\mathbb{Z} /\left(n_{1}+\cdots+n_{k}+1\right) \mathbb{Z}$ is not unique, it is interesting to know which group is indeed obtained in this case. For this, we perform a direct computation for finding a presentation for the fundamental group of the complement of $\tilde{\mathcal{L}}$, using braid monodromy techniques and van Kampen's theorem (for similar computations, see $[1,2,17]$ ). We get that:

$$
\pi_{1}\left(\mathbb{P}^{2}-\tilde{\mathcal{L}}\right) \cong \mathbb{Z}^{m-1} \oplus \mathbb{Z} /\left(n_{1}+\cdots+n_{k}+1\right) \mathbb{Z}
$$

This result is mainly achieved due to the commutative relations induced by the $\left(\begin{array}{c}m \\ 2\end{array}\right)$ nodal points, and the torsion subgroup is created by the projective relation.

\section{An application to Zariski pairs}

As already mentioned, we call a Zariski pair to a pair of plane curves which have the same combinatorics, but their complements are not homeomorphic.

In this short section, we want to use the above constructions to produce new Zariski pairs.

Not every Zariski pair $\left(C_{1}, C_{2}\right)$ can produce a family of Zariski pairs by our construction, since even if $G_{1}=\pi_{1}\left(\mathbb{P}^{2}-C_{1}\right)$ and $G_{2}=\pi_{1}\left(\mathbb{P}^{2}-C_{2}\right)$ are different, it is not guaranteed that $\tilde{G}_{1}=\pi_{1}\left(\mathbb{P}^{2}-\tilde{C}_{1}\right)$ and $\tilde{G}_{2}=\pi_{1}\left(\mathbb{P}^{2}-\tilde{C}_{2}\right)$ will be still different, as there are several ways to construct the same group by central extensions. Therefore, we have to characterize Zariski pairs which induce such families.

A possible characterization is the following:

Proposition 6.1 Let $\left(C_{1}, C_{2}\right)$ be a Zariski pair of two irreducible curves. If $\pi_{1}\left(\mathbb{P}^{2}-C_{1}\right)$ is a cyclic group and $\pi_{1}\left(\mathbb{P}^{2}-C_{2}\right)$ is not a cyclic group, then $\left(\tilde{C}_{1}, \tilde{C}_{2}\right)$ is a Zariski pair too.

Proof Since $\left(C_{1}, C_{2}\right)$ is a Zariski pair, then by definition $C_{1}$ and $C_{2}$ have the same degree and the same singularities. Therefore, using the results of Section 5, $\tilde{C}_{1}$ and $\tilde{C}_{2}$ have the same degree and the same singularities too.

Since $\pi_{1}\left(\mathbb{P}^{2}-C_{1}\right)$ is cyclic and $C_{1}$ is irreducible, then $\pi_{1}\left(\mathbb{P}^{2}-\tilde{C}_{1}\right)$ is cyclic too (by Proposition 4.3). On the other hand, a central extension of non-cyclic group can never be cyclic and hence $\pi_{1}\left(\mathbb{P}^{2}-\tilde{C}_{2}\right)$ is not cyclic. Therefore, $\left(\tilde{C}_{1}, \tilde{C}_{2}\right)$ is a Zariski pair too.

The examples of Zariski [36, 37], Oka [22] and Shimada [27] satisfy the conditions of Proposition 6.1, and hence can be used for producing families of new examples of Zariski pairs. 


\section{Acknowledgments}

I would like to thank Mikhail Zaidenberg and Uzi Vishne for many fruitful discussions. I wish to thank an anonymous referee for improving the paper by his suggestions. Also, I thank Mikhail Zaidenberg and Institut Fourier for hosting my stay.

The author is partially supported by the Chateaubriand postdoctoral fellowship funded by the French government.

\section{References}

[1] Amram, M., Garber, D. and Teicher, M., Fundamental groups of Tangented Conic-Line arrangements I-III, in preparation.

[2] Amram, M. and Teicher, M., Braid monodromy of special curves, J. Knot Theory Ramifications 10(2), 171-212 (2001).

[3] Artal-Bartolo, E., Sur les couples de Zariski, J. Alg. Geom. 3(2), 223-247 (1994).

[4] Artal-Bartolo, E., Fundamental groups of a class of rational cuspidal curves, Manu. Math. 93(3), 273-281 (1997).

[5] Artal-Bartolo, E. and Carmona-Ruber, J., Zariski pairs, fundamental groups and Alexander polynomials, J. Math. Soc. Japan 50(3), 521-543 (1998).

[6] Artal, E., Carmona, J., Cogolludo, J. I. and Tokunaga, H., Sextics with singular points in special position, J. Knot Theory Ramifications 10(4), 547-578 (2001).

[7] Artal-Bartolo, E. and Tokunaga, H., Zariski pairs of index 19 and Mordell-Weil groups of K3 surfaces, Proc. London Math. Soc. (3) 80(1), 127-144 (2000).

[8] Chisini, O., Sulla identità birazionale delle funzioni algebriche di due variabili dotate di una medesima curva di diramazione, Rend. Ist. Lombardo 77, 339-356 (1944).

[9] Degtyarev, A. I., Quintics in $\mathbb{C P}^{2}$ with non-abelian fundamental group, Algebra i Analiz 11(5), 130-151 (1999); Translation in: St. Petersburg Math. J. 11(5), 809-826 (2000).

[10] Flenner, H. and Zaidenberg, M., Rational cuspidal plane curves of type $(d, d-3)$, Math. Nachr. 210, 93-110 (2000).

[11] Fujita, T., On the topology of non-complete surfaces, J. Fac. Sci. Univ. Tokyo Sect. 1A Math. 29, 503-566 (1982).

[12] Garber, D. and Teicher, M., The fundamental group's structure of the complement of some configurations of real line arrangements, Complex Analysis and Algebraic Geometry, edited by T. Peternell and F.-O. Schreyer, de Gruyter, 173-223 (2000). 
[13] Garber, D., Teicher, M. and Vishne, U., $\pi_{1}$-classification of arrangements with up to eight lines, Topology 42(1), 265-289 (2003).

[14] Kulikov, V. S., On Chisini's conjecture, Izv. Ross. Akad. Nauk Ser. Mat. 63:6, 83-116 (1999) [Russian]; English transl., Izvestiya Math. 63, 1139-1170 (1999).

[15] Kulikov, V. S. and Teicher, M., Braid monodromy factorizations and diffeomorphism types, Izv. Ross. Akad. Nauk Ser. Mat. 64(2), 89-120 (2000) [Russian]; English transl., Izvestiya Math. 64(2), 311-341 (2000).

[16] Milnor, J., Morse Theory, Ann. Math. Stud. 51, Princeton University Press, Princeton, NJ, 1963.

[17] Moishezon, B. and Teicher, M., Braid group techniques in complex geometry II, From arrangements of lines and conics to cuspidal curves, Algebraic Geometry, Lect. Notes in Math. 1479, 131-180 (1990).

[18] Nagata, M., On rational surfaces I: irreducible curves of arithmetic genus 0 or 1, Mem. Coll. Sci. Univ. Kyoto Ser. A Math. 32, 351-370 (1960).

[19] Namba, N., Geometry of projective algebraic curves, Pure and Applied Math. 88, Marcel Dekker Inc., 1984.

[20] Oka, M., Two transforms of plane curves and their fundamental groups, J. Math. Sci. Univ. Tokyo 3(2), 399-443 (1996).

[21] Oka, M., Flex curves and their applications, Geom. Dedicata 75(1), 67-100 (1999).

[22] Oka, M., A new Alexander-equivalent Zariski pair, preprint. See: http://www.vkampen.math.metro-u.ac.jp/ oka

[23] Randell, R., The fundamental group of the complement of a union of complex hyperplanes, Invent. Math. 69, 103-108 (1982). Correction, Invent. Math. 80, 467-468 (1985).

[24] Rotman, J. J., An introduction to the theory of groups, Graduate Texts in Math. 148, Springer-Verlag, 1995.

[25] Schafer, J. A., Extensions of nilpotent groups, Houston J. Math. 21(1), 1-16 (1995).

[26] Shimada, I., A weighted version of Zariski's hyperplane section theorem and fundamental groups of complements of plane curves, preprint. See: arXiv:alg-geom/9505004

[27] Shimada, I., A note on Zariski pairs, Compositio Math. 104(2), 125-133 (1996).

[28] Shimada, I., Fundamental groups of complements to singular plane curves, Amer. J. Math. 119(1), 127-157 (1997).

[29] Tokunaga, H., A remark on E. Artal-Bartolo's paper: "On Zariski pairs", Kodai Math. J. 19(2), 207-217 (1996).

[30] Tokunaga, H., Some examples of Zariski pairs arising from certain elliptic K3 surfaces, Math. Z. 227(3), 465-477 (1998). 
[31] Tokunaga, H., Some examples of Zariski pairs arising from certain elliptic K3 surfaces II: Degtyarev's conjecture, Math. Z. 230(2), 389-400 (1999).

[32] Uludağ, A. M., Groupes fondamentaux d'une famille de courbes rationnelles cuspidales, Ph.D. Thesis, Institut Fourier, Universite de Grenoble I (2000).

[33] Uludağ, A. M., More Zariski pairs and finite fundamental group of curve complements, Manu. Math. 106(3), 271-277 (2001).

[34] van Kampen, E. R., On the fundamental group of an algebraic curve, Amer. J. Math. 55, 255-260 (1933).

[35] Zaidenberg, M., Exotic algebraic structures on affine spaces, St. Petersburg Math. J. 11(5), 703-760 (2000).

[36] Zariski, O., On the problem of existence of algebraic functions of two variables possessing a given branch curve, Amer. J. Math. 51, 305-328 (1929).

[37] Zariski, O., The topological discriminant group of a Riemann surface of genus p, Amer. J. Math. 59, 335-358 (1937).

Institut Fourier, BP 74, 38402 Saint-Martin D'Heres CEDEX, FRANCE

Email: garber@mozart.ujf-grenoble.fr

Received: 23 January 2003 Revised: 27 February 2003 